\title{
TelcoFog: A unified flexible fog and cloud computing architecture for $5 G$ networks
}

Authors: Ricard Vilalta (CTTC/CERCA), Victor López (Telefónica), Alessio Giorgetti (Scuola Superiore Sant'Anna), Shuping Peng (Huawei) ${ }^{1}$, Vittorio Orsini (Ericsson), Luis Velasco (UPC), René Serral-Gracià (UPC), Donal Morris (RedZinc), Silvia De Fina (H3G), Filippo Cugini, Piero Castoldi (Scuola Superiore Sant'Anna), Arturo Mayoral, Ramon Casellas, Ricardo Martínez, Christos Verikoukis, Raul Muñoz (CTTC/CERCA).

Abstract: We propose the TelcoFog architecture as a novel, secure, highly distributed and ultradense fog computing infrastructure, which can be allocated at the extreme edge of a wired/wireless network for a Telecom Operator to provide multiple unified, cost-effective and new 5 G services, such as Network Function Virtualization (NFV), Mobile Edge Computing (MEC), and services for third parties (e.g., smart cities, vertical industries or Internet of Things (IoT)).

The distributed and programmable fog technologies that are proposed in TelcoFog are expected to strengthen the position of the Mobile Network and cloud markets. TelcoFog, by design, is capable of integrating an ecosystem for network operators willing to provide NFV, MEC and IoT services. TelcoFog key benefits are the dynamic deployment of new distributed low-latency services.

The novel TelcoFog architecture consists of three main building blocks: a) a scalable TelcoFog node, that is seamlessly integrated in the Telecom infrastructure; b) a TelcoFog controller, focused on service assurance and based on service data modeling using YANG, that is integrated in the management and orchestration architecture of the Telecom operator; and c) TelcoFog services, which are able to run on top of the TelcoFog and Telecom infrastructure. The TelcoFog architecture is validated through a Proof of Concept for loT services.

\section{Introduction}

Telecom operators require cloud computing and storage infrastructures, integrated with their heterogeneous access and transport networks, in order to provide Software Defined Networking (SDN), Network Function Virtualization (NFV), Mobile Edge Computing (MEC), and Cloud Radio Access Network (C-RAN) for future $5 \mathrm{G}$ services. Virtualized functions (e.g., mobile Evolved Packet Core - EPC-, firewall, local cache, video analytics, video storage, central cache, virtual base station, virtualized BBU - BBU hosteling, etc.) are typically deployed in specialized and dedicated hardware. To that end, Telecom operators require to dynamically allocate computing and storage resources to flexibly deploy virtualized functions in cloud infrastructures where needed. Notwithstanding, deploying a private cloud infrastructure integrated with the telecom network presents a differential set of challenges due to the industry's inherent requirements for

\footnotetext{
${ }^{1}$ Shuping Peng was working with Fujitsu Ltd.
} 
high-availability (5-nines), ultra-low latency, and complex networking (Ethernet, optical, wireless, etc.).

Give the tendency to softwarize most the functions of a telecom network, network operators are in transition from operating the network to programming the network. In order to fulfill this vision of intelligent network resources offering NFV, MEC or loT services, spread through the whole network, from the core to the edge, this paper proposes TelcoFog as an innovative architecture based on SDN, NFV and MEC that provides distributed services.

The Fog computing is a relative new cloud computing concept. The basics and objectives of Fog computing (e.g., geographical distribution, low-latency applications, mobility, etc.) have been previously explained in the literature [BoMI-12]. Fog nodes, located at the network edge, are required to support a number of heterogeneous services by providing computational, storage and networking resources. These services have their own requirements in terms of bandwidth, latency, reliability, etc. Moreover, the adoption of Fog computing brings the possibility that (cloud) resources allocated for each service may be distributed among a number of functions (generic virtualized functions) which are instantiated through selected Fog nodes. [OPENFOG] proposes a distributed architecture that solves use cases that cannot be successfully implemented using "cloud only" solutions or independent edge devices.

The distribution of functions and the subsequent forwarding of the (control and data) traffic through those functions is referred to service function chaining (SFC) [HaPi-15]. Within the TelcoFog, the SFC concept is adopted in fog computing, in order to offer added-value services in all the targeted scenarios (i.e., IoT, MEC and NFV).

To make the above concepts reality, it will be necessary to provide a control and orchestration system (the TelcoFog controller) being able to allocate cloud resources for the VNFs of a specific service along with defining its SFC. For the latter, the TelcoFog controller should also ensure the connectivity (network resources) among the different Fog nodes, accommodating the VNFs, to enable traffic steering according to the computed SFC [CTTC_TID_NFV-15]. This provides a seamlessly and unified control for the complete visibility, computation and allocation of both cloud/fog and network resources through different network segments (access, aggregation and transport) assuming heterogeneous access and transport technologies (e.g., Wi-Fi, packet switching, optical transmission, etc.) with the goal of satisfying and ensuring (stringent) service requirements (e.g., zero-latency applications for mission-critical machine communications). To this end, a fundamental action to be done, currently not completely accomplished, is the definition of a common data model, for devices and TelcoFog services that will be used by TelcoFog controller.

Besides the Fog-like nature of TelcoFog and its orchestration capabilities, another critical aspect is the adoption of YANG as a data modeling language, which has been steadily growing in the IT and networking communities for the last few years, for example in optical networking community [CNIT_YANG-16] - and consequently its use has been increasing in multiple standard defining organizations - there is still a lot to define regarding computational resources and devices to cover the loT landscape, from sensors to loT gateways and related applications (such as IoT services and the enabled application ecosystem that better exploit the gathered knowledge) [CLA-16]. Moreover, it shall include smart and expert systems able to apply newer approaches involving the manipulation and processing of a large volume of data (e.g. big data). [OPENFOG] architecture does not include this modelling perspective for services using YANG, 
and thus, it can benefit from TelcoFog innovations. This data modelling approach was introduced by UNIFY Universal Node [UNIFY], while TelcoFog introduces the usage of container technology.

In this context, there are many aspects to standardize including, but not limited to, information and data models for the core/main devices in a given architecture, which can be built by inheritance, or composition, with a common subset, yet allowing for applicable extensions. Another important aspect to standardize are Application Programmable Interfaces (API) between functional elements in the encompassing frameworks and related service workflows, including, as important ones, resource, device, service exposition and (auto)discovery. In line with this, TelcoFog architecture is expected to fulfill the requirements associated to dynamic platforms and deployments, as we demonstrate in the proof of concept (PoC) section (i.e., Section IV).

The adoption of a common, flexible and powerful data and information modeling language to express all sensors, actuators, gateway facilities and services is a first, important step towards the standardization of loT frameworks across multiple vendors beyond the existing ones. Moreover, a standardized loT framework shall include the optimization of the underlying data transport network. Since this objective is known to be ambitious and mid to long term, an important goal is also ensuring at least a certain level of interoperability in complex deployments. The widest adoption of a common set of models, methodologies and approaches is an enabler for a further automation in the integrated control and management of loT platforms that include, in addition to the main components in an loT deployment, the integration of the heterogeneous transport network as a core component.

This paper presents the basic components of the novel TelcoFog system architecture, as well as it provides a PoC for the dynamic deployment of loT services at the network edge. In Section II, this paper introduces the infrastructure challenges from an operator's perspective. Section III focuses on the description of TelcoFog architecture. Section IV introduces the developed TelcoFog PoC, and finally, Section VI concludes.

\section{The challenges for a unified flexible fog computing infrastructure for Telecom Operators}

Telecom operators shall accommodate a wide range of use cases with different requirements (e.g., security, latency, resiliency, and bandwidth) for $5 \mathrm{G}$ services, which will include NFV, MEC or loT services and applications. This opens a whole new set of significant challenges, which we present in this section.

\section{Challenge 1: Fog computing for Network Operators}

It is clear that a distributed data center (DC) architecture is needed for network operators so as to improve the perceived quality of experience (QoE) for their users, reduce energy consumption [UPC_14], or even to have high level and fast reaction in response to events such as network failures. Such distributed cloud architecture was named as the Telecom Cloud in [UPC_TID-15]. Scalability has to be also considered in the telecom cloud since, in contrast to a small number of warehoused-sized DCs commonly used in public clouds, telecom cloud must support a large number of small, distributed DCs to reduce traffic in the core network.

While both, fog and cloud use networking, compute, and storage resources, and share many of the same mechanisms and attributes (virtualization, multi-tenancy) the extension is a non-trivial one. Fog computing enables a new breed of applications and services that goes beyond the 
regular telecom services, so there is a fruitful interplay between the cloud and the fog. Therefore, there is a need for a distributed intelligent platform at the edge that manages distributed computing, networking, and storage resources (see an example in [UPC_TID-15] for video distribution).

\section{Challenge 2: Fog and cloud computing interconnection through operator SDN- enabled networks}

A hybrid cloud-fog computing architecture is needed, which spans from the edge (fog) to the core (cloud). Services deployed in fog nodes will interwork with cloud-located services and thus developing a resource orchestration mechanism will be of the essence. It shall interconnect both fog and cloud domains, which will be interconnected through heterogeneous operator's networks.

A related challenge is the fact that operators are reluctant to incorporate the principles of SDN. There is work to overcome this in [CTTC_TID-16] where the authors propose the usage of a Transport API for the control and management of future transport networks. Even though its detailed definition and development still remain a challenge, it might help the programmability of the operators' SDN-enabled networks. Cloud interconnection has been recently studied [CTTC_TID_SDN-16], but joint integration of fog and cloud paradigms and their network interconnection remains a research challenge. A basic network isolation mechanism at the fog node might be obtained through the introduction of per-tenant software switches, as well as for the TelcoFog network standard isolation mechanisms could be used (e.g., VxLAN, GRE).

\section{Challenge 3: Decentralized NFV services}

The growth of NFV is driving the softwarization of all functions in the network that are traditionally deployed in dedicated hardware. By eliminating specialized network processors, multiple heterogeneous workloads can be consolidated onto a single architecture, thus reducing complexity and simplifying operation, which derives into reducing Total Cost of Ownership (TCO). Therefore, NFV enables the migration into a telecom cloud and the transition of the operator network to software-defined networking (SDN) control, allowing at the same time the programmability of the network resources for fully utilizing the capacity of the deployed physical resources [CTTC_TID_NFV-15].

\section{Challenge 4: Mobile edge computing and D2D communication}

Besides NFV, telecom operators are also studying the benefits and business models of addressing MEC, which provides IT and cloud-computing capabilities within the RAN in close proximity to mobile subscribers. MEC allows content, services and applications to be accelerated, increasing responsiveness from the network edge. The user's experience can be enriched through efficient network and service operations. Using MEC, network operators can deploy fog nodes in the RAN and open the radio network edge to third-party partners, allowing them to rapidly deploy innovative applications and services towards mobile subscribers, enterprises and other vertical segments.

\section{Challenge 5: Smart city services on top of a fog computing architecture}

The need for data processing, analysis, and security close to the connected things at the edge of the network is leading to an explosive growth of independent gateways, repeaters, and systems with vendor's proprietary information models that do not interoperate. This results in siloed solutions which do not interoperate and increase total cost of ownership (TCO) for smart city operators and vertical industries. They must have an open, flexible and secure platform on which 
consolidate siloes and services at the edge and to connect intelligently with the cloud [CISCO15].

\section{Challenge 6: Integration with a big data and analytics framework}

In recent years, the term big data has emerged to describe a new paradigm for data applications. The heart of the big data paradigm is that data is too broad, arrives too fast, changes too fast, contains too much noise, or is too diverse to be processed within a local computing structure using traditional approaches and techniques. Then, the big data paradigm consists of the distribution of data systems across horizontally-coupled independent resources to achieve the scalability needed for the efficient processing of extensive datasets. The challenge is to provide the necessary extensions to big data frameworks in order to provide the necessary TelcoFog node computational and storage resources for applications.

\section{TelcoFog system architecture}

Figure 1 shows the proposed TelcoFog architecture and how it can be introduced at the edge of a Network Operator infrastructure in order to fulfill the presented challenges. We can observe that TelcoFog nodes are placed at the edge of the network. Two scenarios are envisaged: a) integrated network operator services, and b) integrated smart city services. TelcoFog nodes might create an overlay network (referred as TelcoFog edge network) on top of the operator's access network in order to interwork allowing different networking services (such as multicast or anycast services based on the emerging segment routing technology). In both scenarios, the TelcoFog node plays a significant role in providing the necessary distributed and secure computing, storage and networking infrastructure in order to provide the targeted services. The TelcoFog controller can both act in a hierarchical (parent/child) or peer architecture for the optimal allocation of resources across several networks and TelcoFog nodes. It also includes a mechanism for slicing such TelcoFog resources, as well as providing a standardized procedure to describe and deploy TelcoFog services. Finally, to complement these mechanisms, a big data and analytics framework is also provided.

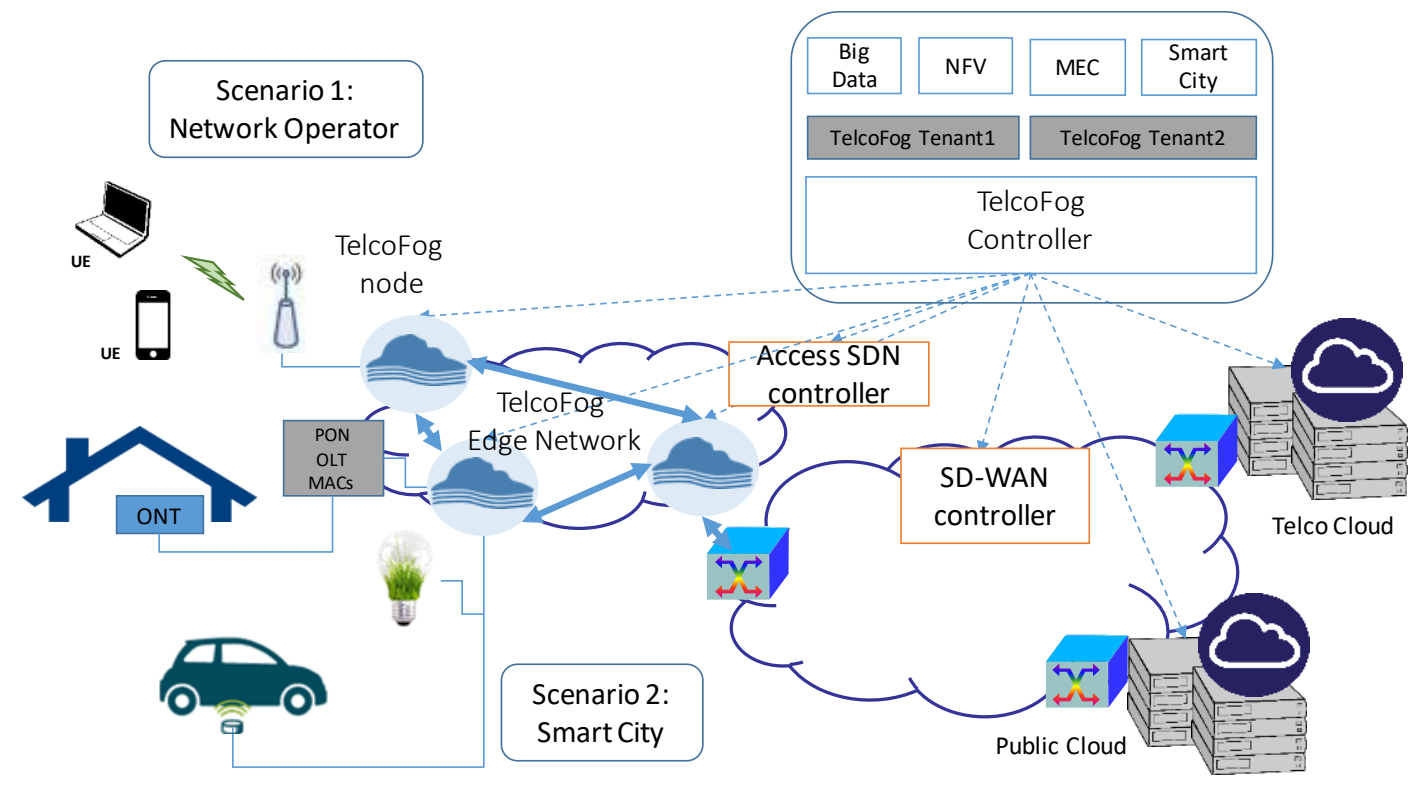

Figure 1 TelcoFog architecture 


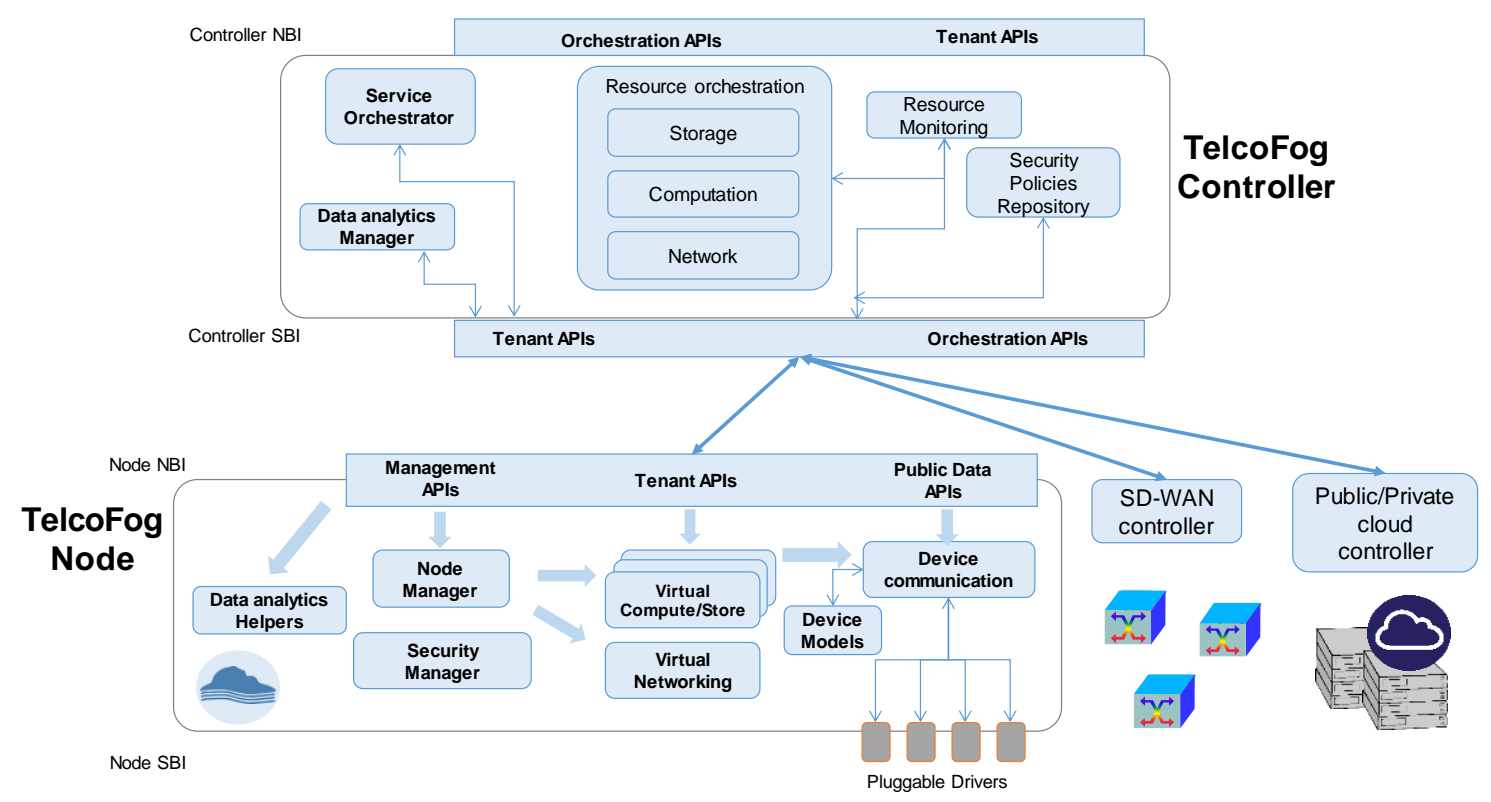

Figure 2 Detailed TelcoFog system architecture

TelcoFog nodes act as interconnected and highly-distributed resources closer to the network edge. To fulfill this view, TelcoFog architecture proposes the necessary technologies to obtain highly performing, secured and resilient TelcoFog nodes, and the necessary TelcoFog controller, which is responsible for the orchestration of TelcoFog resources, as well as their interconnection through highly programmable networks.

Figure 2 provides an overall description of the basic components in both the TelcoFog node (below) and TelcoFog controller (above).

\section{TelcoFog node}

The TelcoFog node is the fog/network infrastructure which is responsible for provisioning the necessary computation, storage and networking resources. Physically, a TelcoFog node, might consist of a set of (mission critical) servers, hard disks and network interface cards. Logically, a TelcoFog node incorporates a node manager in order to handle the offered TelcoFog virtual resources. A Security Manager is the responsible for handling the device authorization, authentication and encryption. Finally, device communication module allows the abstraction and control of the underlying smart things and devices, through the data modeling of the devices. Devices configuration, procedures and notifications are modelled and can be (auto) discovered and related through services running in the TelcoFog node.

The Node Manager handles the optimal allocation of the required virtual resources onto the node physical resources, which are the pool of existing resources within the node. The virtual allocated resources might include containers or virtual machines (depending on the capabilities of the node), allocated virtual memory, virtual disk space or virtual network interface cards and virtual switches. The TelcoFog node leverages on the existing fog node solutions focused on virtual computation resources and it provides the necessary extensions in order to ensure the high degree of resource virtualization.

To access the particular smart things and devices, TelcoFog node foresees the abstraction of the things' data model description by a Device Communication module. Such module provides a set 
of Pluggable Drivers that allow the uniform access to the things, these drivers are extensible and using a public interface to allow the easy integration of sensors, actuators, or any other device by modeling their behavior in the Device Models. This allows the disaggregation of the fog resource towards the extreme edge of the network, as smart devices could also be used for processing, storage or networking purposes. Another important component within the node are the Data Analytics Helpers, a predefined set of functionalities for data analysis and evaluation can be provided as services running on top of allocated virtual resources.

TelcoFog services abstract the access to the different smart things and devices by means of a Device Proxy, as part of the Device Communication module, which interplays between the users, the tenants (through a privileged API allowing an enhanced set of features) and the things. The main purpose of this proxy is to provide a finer granularity and more powerful system to control the access to the things, homogenizing its access, being able to enforce the necessary Security Policies, using the Security Manager to offer the sufficient smart caching capabilities to avoid the unnecessary resource consumption of the things.

Finally, in order to allow the usage of all these functionalities, the TelcoFog node offers a common NorthBound Interface (NBI) that can be split into three different views, i) Management API, ii) Tenant API, iii) Public Data API. The management API is used to manage the resources within the TelcoFog node and should be only used from the TelcoFog Controller (Security Manager enforces this). The Tenant API, is used as a private interface to allow the different tenants to control and manage TelcoFog services, which gather data from the allocated virtual resources. Finally, the Public Data API provides access to the monitored data that Security Manager policy specifies as publicly available.

\section{TelcoFog controller}

The TelcoFog controller extends the concept of an NFV orchestrator in order to support the dynamic deployment of generic virtualized functions (hereafter referred to as TelcoFog services). Its main role is the distributed orchestration of resources and services. To this end, the core block of this unit is the Resource Orchestration, where its main role is to define and enforce the resource orchestration logic, defining the particular logic that allows the system to allocate the different Storage, Computational, and Network resources. To achieve this, the Resource Orchestrator will receive information about the status of each Fog Node through its Resource Monitoring module that will have an updated view of the resource consumption and general status of the system. Besides the pure resource management functionalities, it is also very important to manage the deployment of the different services. This is achieved by using a Service Orchestrator, which at the same time is also in charge of guaranteeing that the service is functioning properly. Finally, to control the access to the different resources, and to define the security policies that will be enforced on each Fog Node, we consider a Security Policies Repository, where the policies are stored and security pushed to the Fog Node.

TelcoFog services are modeled using their YANG data models, and they can be deployed on top of the allocated TelcoFog resources reserved for each tenant. Service Orchestrator is the responsible of requesting the necessary resources and deploying the requested services on top of them. It acts as a generalized NFV Management and Orchestration (NFV MANO), while services are generic virtualized functions, which might or not be network related. It is expected to leverage the work done in NFV and align it with MEC servers, while providing access to the data obtained from the smart things and devices. 
The TelcoFog Resource Orchestrator is the key element to handle the allocation of TelcoFog resources into the deployed TelcoFog nodes, or in centralized Cloud Data Centres.

As previously explained, Resource Monitoring is key in providing Service Assurance, due to the fact that it inspects the provided TelcoFog services in order to anticipate future degradation of the Quality of Experience. Finally, Data analytics Management allows the triggering of specifically configured set of functionalities for data analysis and evaluation can be provided to services running on top of allocated virtual resources.

\section{TelcoFog big data and analytics framework}

One of the main objectives of data analytics is knowledge discovery from data (KDD) [FAY-96]. To that end, the following steps are performed in an iterative way:

- Data pre-processing. Basic operations include data selection (to retrieve data relevant to the KDD task from the database), data cleaning (to remove noise and inconsistent data, to handle the missing data fields, etc.) and data integration (to combine data from multiple sources).

- Data transformation. The goal is to transform data into forms appropriate for the learning and mining task, that is, to find useful features to represent the data. Feature selection and feature transformation are basic operations.

- Data mining and machine learning. This is an essential process where intelligent methods are employed to make predictions and extract patterns (e.g. classification, regression, clustering, etc.).

- Pattern evaluation and presentation. Basic operations include identifying the truly interesting patterns which represent knowledge in an easy-to-understand fashion.

The TelcoFog allows the KDD process previously described that includes data collection, preprocessing and storage, data transformation, and data mining and presentation, so as to offer knowledge as a service to TelcoFog services. The real-time capability will provide mission critical business intelligence in the support of enterprise decision making. Such framework will allow applications to monitor and manage computation and storage resources, while protecting the privacy and integrity of data.

Each component in the fog computing architecture generates detailed event records for every significant event. These event records are kept/sent to one or multiple instances of TelcoFog nodes that collate,store and process the information in a horizontally scalable framework for distributed storage and processing of very large data sets. The nodes include mechanisms to automatically trigger the collection of more detailed records when certain event occurs; the goal is to be able to get to the root cause of any issue without having to reproduce it.

\section{TelcoFog Proof of Concept for IoT services}




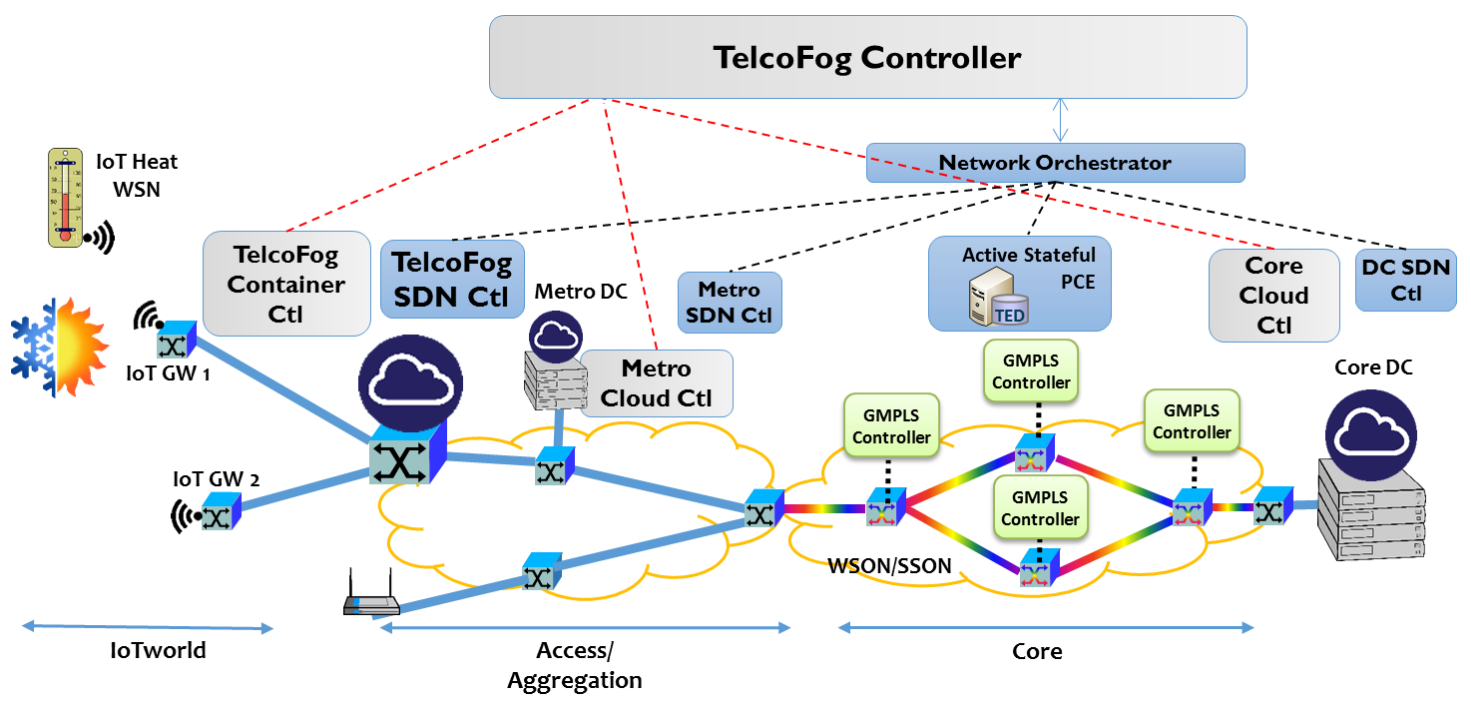

Figure 3 TelcoFog Proof of Concept scenario

IoTWorld Testbed consists of a set of heating, ventilating, and air conditioning (HVAC) modules and actuators. It also includes a WSN, which forward measurements of temperature and energy consumption to an loT gateway. ADRENALINE Testbed is a distributed Cloud Computing platform across access/aggregation and core networks [CTTC_IOT-16].

In this Proof of Concept, shown in Figure 3, we have deployed an SDN/NFV-enabled TelcoFog node in ADRENALINE Testbed and we have interconnected it to loT gateways from the loTWorld Testbed by means of End-to-End (E2E) TelcoFog Controller [CTTC_IOT-16]. Network orchestrator provides network connectivity between loT gateways and deployed containers which might be allocated in the proposed TelcoFog node or towards virtual machines which have been allocated in a DC in the core network. The TelcoFog node has been implemented using Docker containers and OpenVSwitch [TELCOFOG]. An agent allows the dynamic creation of containers and their attachment to the underlying virtual switch. Cloud Controller has been implemented using OpenStack Mitaka.

We have defined a Heating, Ventilation and AC (HVAC) application, which models HVAC services, such as temperature control. The HVAC application is responsible for monitoring temperature sensors and uploading the data into a database running on the cloud. If the application detects an increase of temperature, it is able to activate the AC actuator, in order to reduce the measured temperature. The used YANG model for HVAC agent uses three containers to organize the necessary config/status data for Temperature, Heat and AC. Temperature allows the monitoring of current temperature in Celsius. Heat and AC might be accessed in order to modify the status of the Heat or AC systems (Stop/Run/Error).

The HVAC application is running at the TelcoFog node at the network edge. The location of the application at the network edge improves the latency of the application, and reduces the necessary bandwidth with cloud resources, typically located in distant locations. 


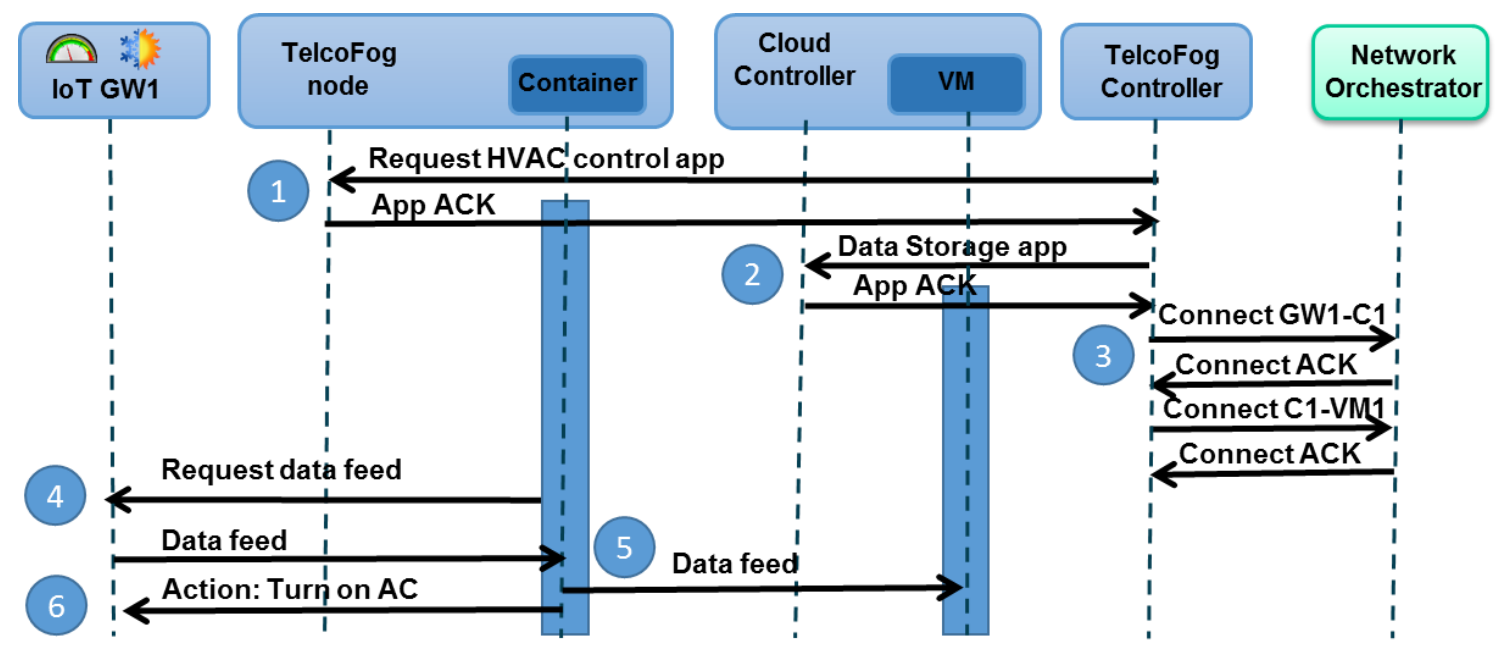

Figure 4 Message Exchange workflow for the deployment and operations of a HVAC application

The TelcoFog Controller is responsible for handling computational and network connectivity requests, which are processed through the Cloud Controller/TelcoFog node and Network orchestrator. The Cloud Controller (OpenStack) is accessed through its REST API, while TelcoFog node and network orchestrator are handled using the YANG-based APIs.

The orchestration process for the deployment of the HVAC application consists of three different steps (Steps 1 to 3) and the HVAC operations include steps 4, 5 and 6 (Figure 4).

a) Step 0: TelcoFog controller is requested to deploy an HVAC service, which will be able to control the temperature locally and store the measurements on a cloud-based database.

b) Step 1: The TelcoFog controller will first request to a Fog node the creation of a container (C1) running the HVAC application.

c) Step 2: Once the container is available, the TelcoFog controller triggers the creation of a database running on a cloud data center (VM1).

d) Step 3: Once the computational resources have been obtained, the TelcoFog controller requests the connectivity services to the network orchestrator. Connectivity services are requested between the HVAC container and the cloud-based database, as well as between the HVAC container and the IoT gateway (GW1).

Once the HVAC service has been fully deployed, the HVAC enters in operation mode.

e) Step 4: the HVAC application continuously monitors the temperature, using a standardized HVAC YANG service.

f) Step 5: The data is stored towards the database running on the cloud.

g) Step 6: If a high temperature is detected, a YANG remote procedure call (RPC) will be triggered towards the IoT GW1, in order to turn on the AC. 


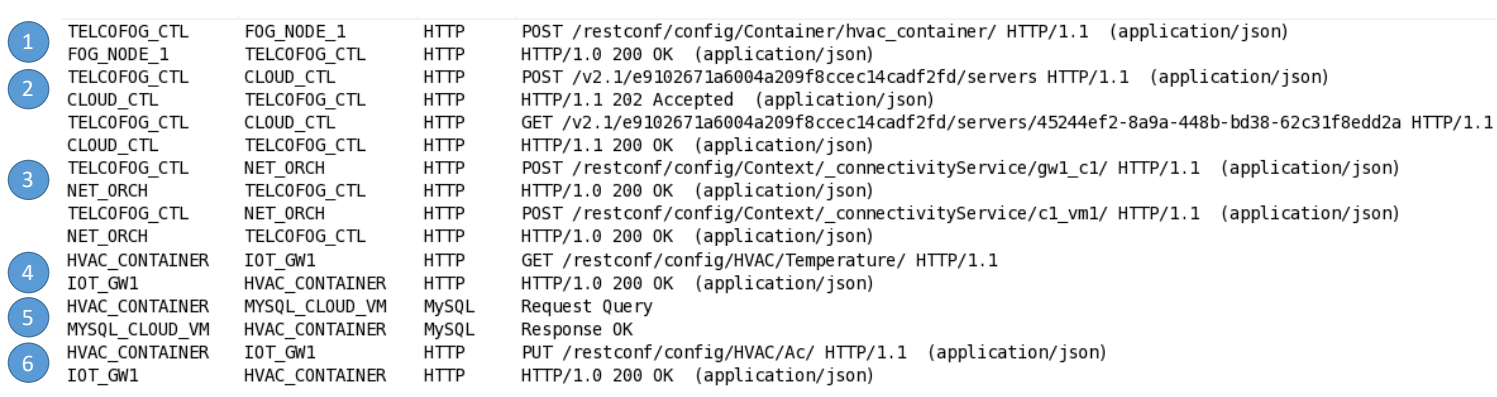

Figure 5 Wireshark capture of the HVAC service deployment and operation

Figure 5 shows the captured Wireshark traces for the TelcoFog orchestration process for HVAC service deployment, as well as the traces from the operation of the HVAC application. It can be observed the usage of RESTconf Protocol for the HVAC, Container and Connectivity Service YANG data models. These Wireshark traces demonstrate the feasibility of the proposed approach, in order to use YANG-based services for both loT and NFV applications.

Table 1 shows the mean delays for 10 orchestration cycles of the proposed experimental Proof of concept. It can be observed that container deployment delay is two orders of magnitude inferior than virtual machine deployment delay, thus allowing faster service deployment. Connectivity services delay is much influenced by the delay between network elements and Network orchestrator, as well as the need for establishment of dynamic optical paths, which increases significantly the setup delay. In case that only edge services are deployed, orchestration delay is reduced to 1.31 seconds.

\begin{tabular}{|l|c|}
\hline $\begin{array}{l}\text { Container deployment delay } \\
\text { Connectivity service setup delay } \\
\text { (GW1 - C1) }\end{array}$ & $399 \mathrm{~ms}$ \\
\hline HVAC command delay & $736 \mathrm{~ms}$ \\
\hline $\begin{array}{l}\text { VM deployment delay } \\
\text { Connectivity service setup delay } \\
\text { (C1 - VM1) }\end{array}$ & $186 \mathrm{~ms}$ \\
\hline $\begin{array}{l}\text { Edge Orchestration delay } \\
\text { Total Orchestration delay }\end{array}$ & $1.36 \mathrm{~s}$ \\
\hline \begin{tabular}{l} 
Table 1 Experimental result for TelcoFog orchestration \\
\hline
\end{tabular}
\end{tabular}

\section{Conclusions}

In this paper we have presented a novel architecture for providing unified cloud and fog resources for deploying NFV, MEC and IoT service on top of a Telecom Operator's network.

A proof of concept for the TelcoFog architecture has been introduced, which focuses on distributed and programmable fog technologies. TelcoFog, by design, is capable of integrating an ecosystem for network operators willing to provide NFV, MEC and IoT services.

The presented HVAC service has demonstrated the feasibility of the proposed approach of using YANG modeling language for IoT services description, as well as the basic for unifying the NFV and MEC services, allowing telecom operators to reduce their TCO.

\section{Acknowledgements}

This work was partially supported by Spanish MINECO project DESTELLO (TEC2015-69256-R). 


\section{References}

[BoMi-12] F. Bonomi, R. Milito, J. Zhu, S. Addepalli, "Fog computing and its role in the internet of things," in: Proceedings of the MCC workshop on Mobile cloud computing, 2012.

[HaPi-15] J. Halpern and C. Pignataro, "Service Function Chaining (SFC) Architecture", IETF RFC 7665, Oct 2015.

[CTTC_TID_NFV-15] R. Vilalta, et al., Transport Network Function Virtualization, Journal of Lightwave Technology, Vol. 33, No. 5, pp. 1-8, April 2015.

[CNIT_YANG-16] Matteo Dallaglio, et al., YANG Model and NETCONF Protocol for Control and Management of Elastic Optical Networks, Optical Fiber Communication Conference, 2016.

[CLA-16] Benoit Claise, YANG as the Data Modeling Language in the IoT space, loT Semantic Interoperability Workshop 2016

[UPC_14] L. Velasco, et al., "Elastic Operations in Federated Datacenters for Performance and Cost Optimization," Elsevier Computer Communications, vol. 50, pp. 142-151, 2014.

[UPC_TID-15] L. Velasco, et al., "A Service-Oriented Hybrid Access Network and Cloud Architecture," IEEE Communications Magazine, vol. 53, pp. 159-165, 2015.

[CTTC_TID_SDN-16] R. Muñoz, et al., Integrated SDN/NFV management and orchestration architecture for dynamic deployment of virtual SDN control instances for virtual tenant networks, Journal of Optical Communications and Networking, Vol. 7, No. 11, 2015.

[CISCO-15] Building Scalable, Sustainable, Smart+Connected Communities with Fog Computing, http://blogs.cisco.com/innovation/barcelona-fog-computing-poc, 2015. Accessed on: 2016/12/22.

[CTTC_IOT-16] R. Vilalta, A. Mayoral, D. Pubill, R. Casellas, R. Martínez, J. Serra, C. Verikoukis, R. Muñoz, End-to-End SDN Orchestration of IoT Services Using an SDN/NFV-enabled Edge Node, in Proceedings of the Optical Fiber Communication Conference and Exhibition (OFC), 20-24 March 2016, Anaheim, California (USA).

[FAY-96] Fayyad, Usama, Gregory Piatetsky-Shapiro, and Padhraic Smyth. "From data mining to knowledge discovery in databases." Al magazine 17.3 (1996): 37.

[CTTC_TID-16] V. López, R. Vilalta, V. Uceda, A. Mayoral, R. Casellas, R. Martinez, R. Munoz, J.P. Fernández-Palacios, Transport API: A Solution for SDN in Carriers Networks, Proceedings of 42nd European Conference on Optical Communication ECOC 2016, Düsseldorf, Germany.

[UNIFY] UNIFY Project, "D5.1: Universal node functional specification and use case requirements on data plane," 2014. Available at: http://www.fp7-unify.eu/files/fp7-unify-eudocs/Results/Deliverables/UNIFY-WP5-D5.1Universal\%20node\%20functional\%20specification.pdf. Accessed on: 2016/12/22.

[TELCOFOG] https://github.com/rvilalta/iotworld. Accessed on: 2016/12/22.

[OPENFOG] OpenFog Consortium Architecture Working Group, OpenFog Architecture Overview, White Paper, February 2016. 